Service social

\title{
Contenu, contexte et enjeux sociaux de la pratique des soins infirmiers à domicile
}

\section{Francine Saillant, Louise Hagan et Gisèle Boucher-Dancause}

Volume 43, numéro 1, 1994

Maintien à domicile

URI : https://id.erudit.org/iderudit/706645ar

DOI : https://doi.org/10.7202/706645ar

Aller au sommaire du numéro

\section{Éditeur(s)}

École de service social de l'Université Laval

ISSN

1708-1734 (numérique)

Découvrir la revue

Citer cet article

Saillant, F., Hagan, L. \& Boucher-Dancause, G. (1994). Contenu, contexte et enjeux sociaux de la pratique des soins infirmiers à domicile. Service social, 43(1), 105-126. https://doi.org/10.7202/706645ar
Résumé de l'article

Cette étude exploratoire a été faite en vue de mieux saisir le contenu, le contexte et les enjeux sociaux de la pratique des soins infirmiers à domicile qui ont pour but de promouvoir l'autonomie des clientèles. Les résultats présentés proviennent d'une analyse thématique du contenu de quinze heures d'entrevue de groupe conduites auprès de six infirmières représentant six des dix CLSC de la région du Québec métropolitain. On observe que la pratique des soins infirmiers s'adresse à une clientèle variée qui vit des situations de soins de plus en plus lourdes. La pratique des soins visant l'autonomie des clientèles doit s'adapter continuellement aux valeurs et au potentiel d'autonomie des personnes et des familles. Divers facteurs personnels et organisationnels limitent ou facilitent la pratique des soins à domicile. Les principaux enjeux sociaux qui marquent la pratique des soins infirmiers à domicile sont soulevés, notamment les nuances à apporter aux construits d'autonomie et de partenariat. 


\title{
Contenu, contexte et enjeux sociaux de la pratique des soins infirmiers à domicile
}

\author{
Francine SAILLANT \\ Professeure-chercheure, École des sciences infirmières \\ Membre du Centre de recherche sur les services communautaires \\ Université Laval \\ Louise HAGAN \\ Professeure-chercheure, École des sciences infirmières \\ Membre du Centre de recherche sur les services communautaires \\ Université Laval
}

Gisèle BOUCHER-DANCAUSE

Diplômée du programme de maîtrise Sciences infirmières, Université Laval Directrice des soins à la Maison Michel-Sarrazin

\section{LES SOINS DANS L'UNIVERS DU SOUTIEN À DOMICILE ET LA QUESTION DE L'AUTONOMIE}

Au cours des récentes années, plusieurs facteurs sont venus bouleverser I'univers des pratiques dans les programmes de maintien à domicile des CLSC. L'un des facteurs qui contribuent le plus à complexifier la tâche des intervenants est sans contredit I'alourdissement de la clientèle. Cet alourdissement est attribuable au vieillissement accéléré de la population et au nombre croissant de personnes très âgées, à l'écourtement des temps d'hospitalisation, aux diverses politiques de 
désinstitutionnalisation, mais aussi à la multiplication des problèmes sociaux engendrés par la pauvreté et la transformation des modes de vie familiaux. La diminution des services hospitaliers entraîne une augmentation de la clientèle postopératoire et de la clientèle aux prises avec des problèmes de santé mentale ou des problèmes psychiatriques. Les personnes souhaitent également de plus en plus mourir à domicile (Roy, Saillant et Vézina, 1994).

La réforme des services sociaux et des services de santé constitue un élément clé du contexte politique dans lequel se situent les services des programmes de maintien à domicile. Cette réforme vise à replacer le citoyen au centre du réseau des services (ministère de la Santé et des Services sociaux, 1991). Parmi les orientations de cette réforme, on trouve celle de promouvoir des services qui se donnent le plus près possible du milieu de vie des personnes et celle de placer le citoyen au cœur de la prise de décision. Les différents services des programmes de maintien à domicile, y compris les soins infirmiers, devront dans cette perspective préserver de grandes forces, telles la promotion de l'autonomie des clientèles et l'approche globale et communautaire. Parmi les objectifs prioritaires à viser, on trouve la prévention de la perte d'autonomie physique, psychologique et sociale, la prévention de l'épuisement familial et l'utilisation optimale des ressources du milieu naturel et des ressources institutionnelles.

On peut s'interroger sur le sort réservé à chacun des acteurs des programmes de maintien à domicile dans le contexte de cette réforme. De l'avis de Jutras et Veilleux (1989), trop de chercheurs se limitent à une identification de partenaires, à une esquisse des dynamiques à soutenir et à des arguments rhétoriques. De plus, l'insistance sur les valeurs liées à l'autonomie dans ces programmes n'est pas sans poser problème face aux clientèles lourdes, et les limites du partenariat apparaissent difficiles à établir. Le citoyen de la réforme est un citoyen idéal : libre de décider et de faire des choix, engagé, il participe dans la "cité des services»; on le veut partenaire, autonome le plus possible par rapport à l'État, n'entretenant pas la dépendance (Saillant, 1992). Ce citoyen idéal correspond de moins en moins à celui que I'on rencontre le plus souvent dans les services de maintien à domicile. II existe bien entendu des degrés à l'autonomie, et chaque citoyen, même gravement malade, conserve un potentiel d'autonomie, si minime soitil. Cependant, "liberté », " autonomie», "participation » et "partenariat» ne sont pas toujours au rendez-vous. Le schizophrène de 35 ans qui vit avec sa mère, la femme âgée qui présente des troubles cognitifs sévères et dont son conjoint et une de ses filles s'occupent, le sidéen qui a choisi de mourir à domicile sont parmi les personnes qui ne présentent pas toutes les caractéristiques de cet idéal. 
Quant aux intervenants, qui sont à la fois promoteurs et véhicules de la réforme, ils doivent sans cesse mesurer la quantité de services, $c^{\prime}$ est-à-dire prodiguer un maximum de services pour un maximum de personnes, dans un État qui exige rationalisation et économie. L'univers des pratiques dans les programmes de maintien à domicile se définit notamment par divers types d'intervention professionnelle. L'un des secteurs où le volume de pratique est très important est celui des soins. Compte tenu de la prépondérance des problèmes de santé physique et mentale qui affectent cette clientèle, celle-ci nécessite très souvent des soins généraux et spécialisés plus ou moins intenses.

Dans la pratique des soins infirmiers, la promotion de l'autonomie des personnes consiste en principe à contribuer efficacement à maintenir le plus longtemps possible les personnes dans leur milieu de vie malgré la présence d'un problème de santé ou d'un handicap. Ici, «le plus longtemps possible» peut refléter le souhait des bénéficiaires d'éviter l'institutionnalisation, mais il faut se demander également quelles sont les implications et la signification de cette insistance sur l'autonomie des clientèles et sur la pratique des soins infirmiers. L'autonomie est-elle un objectif souhaitable en toutes circonstances, pour toutes les catégories de bénéficiaires? Le partenariat souhaité comme moyen de favoriser l'autonomie est-il un facteur d'alourdissement des tâches pour les infirmières et les familles? Enfin, et surtout, quelles sont les réalités de l'autonomie et du partenariat dans la pratique quotidienne des infirmières qui participent aux programmes de maintien à domicile des CLSC? Quels en sont les enjeux sociaux? Dans la mesure où l'on connaît peu de chose de l'univers des soins infirmiers à domicile, qui constitue par ailleurs un secteur de choix pour l'observation de la rencontre du social et de la santé, l'exploration du contenu, du contexte et des enjeux sociaux qui traversent ces pratiques professionnelles apparaît comme l'étape préliminaire d'un questionnement plus vaste portant sur la dynamique du partenariat et du partage des responsabilités de soins entre infirmières et usagers dans les programmes de maintien à domicile des CLSC.

\section{LES SOINS INFIRMIERS À DOMICILE: UN UNIVERS MÉCONNU}

Les publications qui traitent des soins infirmiers à domicile décrivent pour la plupart les grandes orientations de la pratique des soins à domicile et quelques publications font état des caractéristiques du rôle de l'infirmière dans ce domaine (Wiles, 1984; Collière, 1986; Johnson et Jackson, 1989; Pesznecker et al., 1989; Acree et Gregory, 1989; McMahon, 1990; Kristjanson et Chalmers, 1990 ; Kerkstra et al., 1991 ; Horning, 1991). Un nombre restreint d'études présentent la pratique 
des soins à domicile dans une perspective historique (Whall, 1986; Hamilton, 1988). Nous avons recensé une étude de nature évaluative qui traite spécifiquement de la perception des clients quant à l'autosoin dans les services de soins à domicile en Hollande (Van Agthoven et Plomp, 1989). Cette étude fait ressortir le fait que les familles souhaitent en général obtenir de l'aide des infirmières surtout pour développer des habiletés de soins, afin d'aider un proche sur le plan des besoins physiques, alors que pour les besoins de nature psychologique elles souhaitent davantage traiter de ces problèmes entre les membres de la famille sans nécessairement recourir aux soins infirmiers. Nous n'avons relevé aucune étude qui décrit spécifiquement la nature ou l'efficacité des soins infirmiers reliés à la promotion de l'autonomie de la clientèle de soins à domicile.

Les recherches québécoises récentes sur le soutien à domicile portent surtout sur le volet des services informels de soutien à domicile au Québec et sur les rapports entre bénéficiaires et aidants naturels. Elles documentent les questions relatives aux caractéristiques des clientèles cibles et des personnes-soutiens (Fédération des CLSC, 1986; Therrien, 1987 ; Jutras et Renaud, 1987 ; Lesemann et Chaume, 1989; Jutras et Veilleux, 1989; Garant et Bolduc, 1990 ; Roy, Vézina et Paradis, 1992). Toutes ces études mettent l'accent sur le fardeau des aidants naturels dans le contexte des divers programmes de soutien à domicile mis en place par l'État dans les années 80. À notre connaissance, deux études seulement, l'une québécoise, celle de Guberman, Maheu et Maillé (1991), et l'autre américaine, celle de Glazer (1990), montrent que le fardeau qui incombe actuellement aux aidants naturels relève fréquemment de tâches spécialisées, à caractère professionnel, notamment du domaine des soins infirmiers. L'univers du soutien à domicile est donc assez méconnu en ce qui concerne les soins proprement dits.

\section{UNE ÉTUDE EXPLORATOIRE SUR LES SOINS INFIRMIERS À DOMICILE EN CLSC}

Une étude exploratoire ${ }^{1}$ a été menée afin de mieux cerner le contenu, le contexte et les enjeux de la pratique des soins infirmiers à domicile en CLSC.

1. Cette étude exploratoire a été menée à l'École des sciences infirmières de l'Université Laval et au Centre de recherche sur les services communautaires (CRSC) de l'Université Laval. Elle a été soutenue par le Bureau de soutien à la recherche (BSR) et constitue la première phase d'une étude plus vaste subventionnée actuellement par le Conseil québécois de la recherche sociale (CQRS) (EA-333 092). Ces travaux s'inscrivent dans 
Nous voulions ainsi mieux connaître:

1) Les situations cliniques les plus courantes où l'autonomie des clientèles constitue le but visé par la prestation des soins infirmiers, de même que la nature des interventions de soins infirmiers dont le but est de promouvoir l'autonomie des clientèles (contenu);

2) les facteurs personnels et organisationnels qui, de l'avis des infirmières, influencent (facilitent ou limitent) la pratique des soins infirmiers à domicile visant la promotion de l'autonomie des clientèles (contexte);

3) les éléments problématiques des soins infirmiers à domicile par rapport à l'évolution actuelle des programmes de maintien à domicile (enjeux sociaux).

Cette étude exploratoire s'est déroulée entre septembre 1992 et février 1993. L'ensemble des CLSC du Québec métropolitain $(N=10)$ a été sollicité pour la participation des infirmières à cette étude exploratoire. Six infirmières volontaires représentant six des dix Centres locaux de services communautaires (CLSC) du Québec métropolitain ont été recrutées par leur CLSC pour participer à l'étude. Parmi les territoires desservis par les CLSC participants, on trouvait des milieux urbains, semi-ruraux et ruraux couvrant des secteurs favorisés et défavorisés. Le groupe d'infirmières représentait la pratique des soins infirmiers dans des CLSC desservant des populations variées sur le plan socio-économique et sur le plan des profils de santé. Ces six infirmières possédaient entre 10 et 23 ans d'expérience dans les services de soins à domicile. Une infirmière détenait un diplôme de premier cycle en sciences infirmières et les autres une formation collégiale ou l'équivalent. Nous avons jugé que des infirmières longuement expérimentées constituaient des informatrices idéales pour décrire leur pratique, son contexte et ses enjeux, ayant à la fois à leur crédit le vécu des changements opérés dans les programmes de soutien à domicile, une bonne connaissance de leur clientèle et un potentiel d'analyse approfondie de leur pratique par la voie du partage des connaissances que supposaient les rencontres de groupe que nous avions planifiées.

une programmation de recherche dirigée par Francine Saillant et Aline Vézina du CRSC, portant sur la dynamique des acteurs du milieu naturel et des services des programmes de soutien à domicile des CLSC, laquelle est subventionnée par le CQRS (RS-2101 092). Les auteures tiennent à remercier vivement les infirmières participantes pour leur intérêt aux rencontres de groupe qui ont fourni la base de l'information contenue dans cet article. Soulignons que L'infirmière du Québec traite d'autres aspects de cette recherche (Hagan, Saillant et Boucher-Dancause, 1994). 
Une stratégie de collecte de données inspirée des groupes de discussion (focus groups) ${ }^{2}$ a été utilisée. Cette méthode qualitative permet de cerner les dimensions de phénomènes peu explorés en donnant libre cours aux clarifications et à la libre expression de sujets au cours de rencontres de groupes (Gilmore et al., 1989). De notre point de vue, cette méthode s'appliquait bien à une exploration du contenu, du contexte et des enjeux de la pratique actuelle des soins infirmiers à domicile.

Cinq rencontres ont eu lieu, d'une durée totale de quinze heures, espacées à toutes les trois ou quatre semaines. La participation des infirmières a été assidue et leur intérêt a été marqué tout au long des discussions. Ces rencontres ont été enregistrées sur bandes vidéo avec le consentement des participantes.

Une synthèse thématique a été effectuée après chaque rencontre et un rapport synthèse a été rédigé et communiqué aux infirmières participant à l'étude au moment de la dernière rencontre, afin de valider le contenu. Le contenu du rapport a été légèrement révisé en fonction des commentaires reçus de la part des participantes. Le matériel a été soumis à une analyse qualitative de contenu ${ }^{3}$. Une définition consensuelle de l'autonomie, en relation avec la pratique des soins infirmiers à domicile, a aussi été produite ${ }^{4}$.

\section{CONTENU ET CONTEXTE DE LA PRATIQUE DES SOINS INFIRMIERS À DOMICILE}

Dans les sections qui suivent, nous décrivons et analysons le matériel recueilli au cours des entrevues. Le contenu est présenté en relation avec

2. Le groupe de discussion (focus group) est l'une des méthodes souvent utilisées pour la cueillette de besoins. Dans son usage habituel, les utilisateurs échantillonnent, sur une base non probabiliste, des groupes qu'ils rencontrent une ou plusieurs fois. Les groupes retenus et les individus qui les composent doivent représenter les propriétés et caractéristiques d'une population. La technique d'animation est celle de l'entrevue centrée, appliquée à un groupe. Dans le contexte de cette étude, nous avons choisi de ne constituer qu'un seul groupe, composé d'individus devant représenter la variété des contextes de pratique de soins infirmiers à domicile des CLSC du Québec métro. Étant donné la richesse du contenu à explorer, nous avons choisi d'effectuer plusieurs rencontres.

3. Chaque entrevue de groupe a été animée d'après des questions ouvertes soumises au début de chaque rencontre. Après chaque séance, le matériel vidéo a été visionné et le contenu verbal a été synthétisé en fonction des questions autour desquelles la discussion avait eu lieu. Les cinq rapports synthèses constituent la base du matériel d'analyse de contenu.

4. Voici la définition qui a été produite : «capacité du bénéficiaire à définir son niveau de responsabilité et à participer à la recherche d'aide lorsque ses limites personnelles l'exigent ». 
les trois thèmes que nous cherchions à documenter. Les discussions ont porté essentiellement sur le contenu et le contexte des soins infirmiers à domicile visant, en principe tout au moins, la promotion de l'autonomie. Le contenu des soins infirmiers à domicile sera abordé sous l'angle des situations cliniques courantes, de la définition de l'autonomie et des fonctions qui lui sont associées, puis des caractéristiques des pratiques.

Nous explorerons le contexte des soins par le biais des facteurs personnels ou organisationnels qui facilitent ou entravent les pratiques de soins. Les enjeux sociaux des soins seront ensuite dégagés des éléments de contenu et de contexte discutés par les infirmières participantes.

\section{Le contenu}

La promotion de l'autonomie des clientèles dans la pratique des soins infirmiers à domicile: une brève description des situations cliniques les plus courantes

La prestation des soins infirmiers à domicile dans les CLSC visés par l'étude s'adresse à une clientèle en majorité adulte et plutôt âgée, aux prises avec des problèmes de santé assez variés dont les plus courants sont le diabète, les problèmes cardiaques, les problèmes respiratoires chroniques, différentes formes de cancer et les accidents cérébrovasculaires. Les soins visent surtout à développer les capacités des personnes et des proches à maintenir ou à améliorer l'intégrité physique structurelle et fonctionnelle. Les besoins d'ordre psychosocial, reliés à l'altération de la santé physique des bénéficiaires, sont également souvent l'objet des soins infirmiers pour aider les personnes à s'adapter à leur condition de malades et à adapter l'environnement à cette condition.

Le besoin de développer la compétence du bénéficiaire et des proches en vue d'une autonomie optimale s'évalue selon les infirmières par l'observation des limites ou des incapacités chez ces personnes à assumer le dépistage, l'observation et le traitement de certains symptômes ou complications d'une maladie. C'est le cas, notamment, de I'observation et du soin des plaies consécutives à une chirurgie ou à une complication d'un diabète ou d'un autre problème du système circulatoire. La prise de médication plus ou moins fidèle sur le plan thérapeutique médical ainsi que la surveillance plus ou moins suivie et systématique des signes et symptômes de complications des maladies sont également des indices du besoin de développer la compétence des personnes concernées. Il y a aussi les difficultés plus techniques, telles que les soins associés à l'entretien d'une sonde vésicale (les irrigations vésicales), les techniques d'hygiène d'une stomie, les prélèvements sanguins par micro-méthodes pour le contrôle du diabète. 
Selon les infirmières, il y aurait une part importante de temps consacrée aux soins palliatifs, notamment au développement des habiletés des proches à assurer le confort physique et psychologique de la personne mourante, à contrôler la douleur et les effets secondaires des anti-néoplasiques ou autres médications.

\section{La promotion de l'autonomie: des fonctions variées, une définition nuancée}

Les discussions entourant le thème de l'autonomie et sa signification dans le contexte des soins infirmiers à domicile ont été fécondes et montrent, de façon générale, des vues très nuancées par rapport au modèle normalisateur proposé par l'État. L'autonomie, tout en étant un idéal, ne peut être un objectif universel pour tous.

En premier lieu, les infirmières décrivent les fonctions visant la promotion de l'autonomie comme étant celles qui concernent plus spécifiquement le domaine de l'éducation pour la santé ou de l'enseignement individuel du bénéficiaire ou de ses proches, mais aussi du réseau naturel. Les activités éducatives ont pour but de favoriser leur participation dans le traitement médical des problèmes de santé physique, dans les soins de maintien de l'intégrité physique, et dans la recherche de mécanismes d'adaptation à la maladie ou à un handicap; elles peuvent aussi contribuer à favoriser la création d'un environnement social et physique adapté à la condition du bénéficiaire. Enfin, le soutien et le renforcement des comportements ou des attitudes du bénéficiaire et de ses proches, par le moyen de l'écoute active, de la relation d'aide, ou encore par le recours au soutien matériel et à l'organisation des services seraient aussi des fonctions visant la promotion de l'autonomie.

Les fonctions infirmières orientées vers la promotion de l'autonomie sont variées; derrière la description sommaire de ces fonctions, quelles conceptions les infirmières se font-elles de cette notion de "promotion de l'autonomie»?

L'autonomie a été définie par les infirmières participantes comme étant la capacité qu'a le bénéficiaire de définir son niveau de responsabilité et de participer à la recherche d'aide lorsque ses limites personnelles l'exigent. Comme le souligne l'une d'elles, c'est:

Quand je suis capable de déterminer son degré de responsabilité et puis sa possibilité de réaction à l'égard de la situation qu'elle vit. Peut-être qu'elle n'est pas capable elle-même de faire le geste nécessaire pour se sortir d'une situation, mais elle se montre capable de penser à faire des démarches ou de penser à quelque chose la concernant, pour améliorer son cas. Je pense que cette personne a une certaine autonomie, parce 
qu'elle ne se laisse pas aller et qu'elle fait un peu l'effort de trouver une solution.

L'autonomie fut aussi discutée par les infirmières comme étant la capacité d'une personne à maintenir une qualité de vie conforme à ses valeurs et à ses besoins et ce, avec ou sans aide extérieure. L'autonomie devient donc, dans ce contexte, la capacité de subvenir à ses besoins globaux, en tenant compte de ses limites. Une personne est autonome dans la mesure où elle peut assumer elle-même, ou avec I'aide de son réseau naturel, les activités de la vie quotidienne (AVQ) et les activités de la vie domestique (AVD) ${ }^{5}$. Les citations suivantes illustrent ce point de vue:

C'est la capacité d'une personne d'avoir une qualité de vie dans son milieu, peu importe où est son milieu, avec ou sans aide extérieure, une personne qui répond à ses besoins et à ses valeurs à elle, même si ça ne répond pas à mes propres idéaux d'autonomie.

$C^{\prime}$ est une personne qui a des pertes, des limites, mais qui accepte I'aide et qui va la chercher; notre rôle est de développer au maximum le potentiel qui lui reste. Par exemple, dans le cas d'un hémiplégique qui n'est pas capable de se servir de son bras droit, je dois maximiser l'usage de son bras gauche et ne pas faire à sa place tout ce qu'il est capable de faire seul; ça m'apparaît à moi très important. Et «travailler» aussi le réseau.

L'autonomie dans le contexte des soins infirmiers à domicile n'est donc pas le fait pour un bénéficiaire d'assumer entièrement les soins requis par sa condition physique ou sociale. Elle reflète plutôt une reconnaissance par ce dernier de ses limites personnelles ainsi qu'une volonté et une capacité de contribuer à la recherche de solutions pour compenser ces limites. Il y a donc implicitement le respect du potentiel des personnes.

Il est possible qu'on n'accepte jamais d'avoir des limites, mais on peut accepter de vivre avec et essayer de développer une façon d'être autonome.

Promouvoir l'autonomie dans le contexte des soins infirmiers à domicile signifie donc impliquer la personne dans la solution de son problème de santé en maximisant son potentiel. C'est aussi largement chercher la participation des membres du réseau naturel lorsque ce réseau existe, mais la promotion de l'autonomie doit se faire, selon les infirmières participantes, en tenant compte des valeurs et des limites des bénéficiaires et des familles.

5. Activités de la vie quotidienne (s'habiller, se laver, se nourrir, etc.). Activités de la vie domestique (entretien ménager, cuisine, etc.). 


\section{Les caractéristiques des soins infirmiers visant l'autonomie des bénéficiaires à domicile}

La promotion de l'autonomie par la prestation des soins infirmiers a été analysée pour chacune des étapes de la démarche de soins, notamment la définition des besoins et des priorités de la clientèle, le choix des interventions de soins dans le contexte des activités de nature éducative, et enfin, les moyens de soutien généralement mis en œuvre, dont la relation d'aide.

La définition des besoins se fait au moment de la visite initiale, mais aussi à chacune des rencontres entre l'infirmière et le bénéficiaire dans le contexte du processus de la relation d'aide inhérente à la démarche éducative. Ces besoins peuvent être variés et ils ont souvent un caractère imprévisible; ils peuvent se révéler différents de ceux qui ont initialement motivé la visite de l'infirmière:

Même si je suis partie du bureau le matin avec une idée en tête ( "ce matin je lui parle de ça»), j'arrive, et la veille sa fille a eu quelque chose, un gros problème conjugal. Elle est préoccupée par ce problème, sa glycémie est très haute. Je vais laisser tomber ma priorité, qui pourtant était si importante en partant du bureau, et on va s'occuper de ça, je vais l'écouter. Je vais passer peut-être une heure à l'écouter, mais elle avait juste besoin de parler de ça ce matin-là. Je ne fais donc ni enseignement ni rien d'autre.

Ces besoins peuvent être reliés à l'acquisition d'habiletés techniques, mais ils dépassent la plupart du temps ce niveau, notamment chaque fois que l'infirmière intervient dans le sens de l'aide au développement de mécanismes d'adaptation à l'égard de la maladie chronique, pour la personne atteinte aussi bien que pour la famille.

L'aide apportée au processus d'adaptation à la maladie implique directement la promotion de l'autonomie, laquelle n'est pas sans engendrer des dilemmes éthiques pour les infirmières. Celles-ci ont mentionné l'existence de conflits entre le désir de l'intervenant ou de l'intervenante de promouvoir l'autonomie et celui du bénéficiaire qui peut parfois résister à s'impliquer dans la solution de son problème. L'une des infirmières rend parfaitement compte de ce type de dilemme, lié à des valeurs non partagées, fréquemment vécu dans les soins à domicile.

...des gens qui ne veulent avoir d'aide de personne. Il y a une dame et un homme qui vivent dans un état d'insalubrité tel que $s^{\prime} i l$ fallait que des journalistes ramassent ça, ils diraient à quel point ça n'a pas de bon sens. Tu te pinces le nez et tu t'arraches le cœur chaque fois que tu entres chez eux. Ils ne veulent rien faire. Mais avec cette espèce de fameuse charte des droits et libertés des gens, tu ne peux plus forcer personne. 
Même avec une évaluation psychiatrique qu'on a fait faire dans le cas d'une personne, le médecin l'a renvoyée et a dit: il est capable de décider pour lui. Par contre, ç’a été accepté par la curatelle: il n'était pas capable de gérer ses biens. Alors, il y a comme deux mesures, et tu te dis: Qu'est-ce que je fais dans ce cas-là? Est-ce que je vais les laisser là ? Par exemple, c'est quoi la notion d'autonomie sur le plan mental ? C'est quoi, es-tu capable de choisir ce qui est bon pour toi, et à la limite jusqu'où ça va ce qui est bon et ce qui n'est pas bon? Arrêtestu de donner tous les soins? C'est arrivé dans certains dossiers qu'on s'est complètement retiré jusqu'à ce que ça éclate et qu'il y ait une hospitalisation, et que la personne se rende compte, plus ou moins, qu'elle avait besoin qu'on la soutienne. C'est quoi l'autonomie? Quand c'est physique, c'est un peu plus clair, mais quand c'est psychologique, comportemental, ça ne se tranche pas au couteau comme ça. T'as les valeurs, mais il y a comme une limite.

La définition des besoins de soins ne va pas sans l'établissement de priorités de soins pour la clientèle. Elle se fait d'abord en fonction du risque pour la survie du bénéficiaire et de ses proches ainsi que du niveau d'anxiété ressenti par ces derniers.

Les infirmières fixent les objectifs du niveau d'autonomie à atteindre en fonction du potentiel spécifique du bénéficiaire ainsi que de ses limites personnelles et des limites environnementales (physiques et sociales). On observe une tendance à l'individualisation des soins selon les besoins des bénéficiaires. Pour cette raison, le délai pour I'atteinte des objectifs d'autonomie demeure souvent imprévisible et variable selon les contextes.

Des fois il y a des problèmes d'acceptation. Je me souviens $d^{\prime}$ une cliente. Seulement pour lui donner son insuline, je pense qu'il a fallu y aller pendant deux mois, parce qu'elle ne voulait rien savoir de l'insuline. Après quelques visites, on se rend compte qu'on travaille seulement là-dessus. On a été obligé de laisser tomber le reste de l'enseignement: $y^{\prime}$ a rien qui rentrait. Une fois qu'elle s'est aperçue que c'était pas si mal, qu'elle a fait un bout de chemin par rapport à l'acceptation, on a pu commencer à aller au niveau des connaissances et des savoirs. C'est un élément bien important, parce qu'on peut parler longtemps dans le vide si les gens sont en période de négation par rapport à quelque chose, que ce soit un problème cardiaque, une amputation, une colostomie, le diabète. II faut vraiment tenir compte de ça.

Par ailleurs, les activités de soins sont en général plus intenses et plus fréquentes au début du service, parce qu'elles ont alors plus 
d'impact pour réduire l'anxiété et créer un climat de confiance. De plus, le délai est également fonction de l'âge, de la condition de santé de la personne et de son niveau de confiance en ses capacités personnelles.

En ce qui concerne les interventions de soins de nature éducative, les infirmières disent vérifier $\mathrm{d}^{\prime}$ abord le niveau des connaissances ou des habiletés afin d'évaluer les besoins d'apprentissage du bénéficiaire. L'infirmière explore aussi l'univers émotif associé à la maladie ou au traitement. À cette occasion, elle décode les émotions en établissant des liens avec les symptômes ressentis par la personne, par exemple au moment des épisodes d'hypo ou d'hyperglycémie.

Les moyens pédagogiques utilisés sont de nature plutôt informelle et de type échange-discussion avec les bénéficiaires et les proches. Lorsque les proches participent aux activités éducatives, I'implication est perçue par les infirmières comme étant facilitante pour promouvoir l'autonomie du bénéficiaire. Elle constitue, par ailleurs, parfois un fardeau de tâche supplémentaire, puisque ces personnes ont également des besoins qui leur sont particuliers.

Les infirmières établissent à l'occasion un contrat explicite avec les bénéficiaires; ce contrat peut contenir les conditions du partage des responsabilités de soins et des rôles entre celles-ci et le bénéficiaire. Cette approche éducative sous forme de contrat explicite n'est cependant pas une pratique courante. Elle est en général utilisée sous forme verbale et elle est révisée régulièrement.

Le soutien à l'éducation et à la promotion de l'autonomie passe nécessairement par un lien médiateur basé sur les principes de la relation d'aide. La reconnaissance des limites du bénéficiaire, de sa souffrance et l'importance du temps accordé à chaque personne sont déterminantes. Sans la confiance mutuelle, la promotion de l'autonomie demeure un principe creux et sans portée réelle. De façon générale, les infirmières sont d'avis que la base de la relation de confiance qui s'établit entre elles et les bénéficiaires repose sur le fait qu'elles sont perçues comme des personnes non menaçantes et sécurisantes. Le «corps » constitue la voie d'accès à l'établissement de la relation d'aide, puisque la très grande majorité des demandes de soins infirmiers à domicile sont motivées initialement par un problème de santé physique.

$C^{\prime}$ est souvent qu'on a des cas comme ça où, à travers un problème de santé, il va se passer beaucoup d'autres choses. Je pense que ce qui est spécifique à nous, c'est qu'on est capables de faire le lien ; derrière un problème de santé quelconque, il y a probablement autre chose. $C^{\prime}$ est pas seulement le problème de santé, y'a autre chose en dessous de ça. À cause de notre expérience et de notre formation on est capables d'aller chercher d'autres éléments pour les faire débloquer sur autre chose. 
Je pense qu'il y a un aspect qui est moins menaçant, on va écouter davantage, "tolérer » davantage sans obliger la personne à avoir des changements de comportements.

Cette relation d'aide s'établit dans la mesure où l'infirmière prend le temps d'écouter, de laisser s'exprimer les émotions, manifestant un intérêt pour la personne. Elle fait en sorte que le bénéficiaire n'ait pas I'impression qu'elle est pressée par le temps, contribuant ainsi à réduire I'anxiété. Cette relation d'aide équivaut à une forme d'accompagnement de la personne dans sa souffrance et dans sa détresse face à la perte de ses capacités d'autonomie.

Certaines situations de soins sont jugées plus lourdes dans ces formes d'accompagnement. L'expérience du cancer, notamment, exige un plus grand engagement affectif de la part des infirmières et requiert une plus grande disponibilité auprès du bénéficiaire et des membres de la famille.

Moi, je pense qu'on reçoit beaucoup quand même du client en même temps. Bon, ça reste que pour nous aussi, parfois, ça peut être lourd à porter. Lourd, dans le sens que, bon, tu ne restes pas neutre face à des situations comme ça. II nous faut ventiler nous aussi. Moi, je pense qu'on reçoit énormément. $C^{\prime}$ est très valorisant.

Malgré la lourdeur de la tâche, la relation d'aide est aussi vécue comme un important facteur de motivation, comme une source de valorisation, un gage de réciprocité, mais aussi une occasion de croissance lorsqu'il y a prise de conscience de la souffrance humaine et du courage des bénéficiaires en comparaison avec la condition personnelle de l'infirmière. Ces situations, bien qu'elles soient difficiles parfois, sont sources de réflexion et riches de sens sur le plan spirituel.

Moi je dis que quand ils nous mettent dans un état d'âme, qu'on est là avec eux et qu'on les écoute, à un moment donné on a l'impression d'être rempli de quelque chose, juste à les écouter. Ils viennent vivre avec toi en dedans quelques minutes. Tu les rejoins quelque part.

Deux caractéristiques des soins infirmiers à domicile se sont dégagées des rencontres: la première est celle de l'individualisation des besoins et des priorités de soins, la deuxième est celle de la valorisation de l'accompagnement et des liens dans la relation d'aide.

\section{Les facteurs qui influencent la pratique des soins infirmiers à domicile : le contexte}

Les infirmières ont reconnu que les facteurs personnels, telles les croyances, les attitudes et les valeurs, et certaines méthodes de travail ou 
encore des facteurs organisationnels ont un impact sur la pratique des soins infirmiers à domicile. Certains de ces facteurs ont été évalués en fonction de leur rôle facilitateur ou comme éléments de motivation, tandis que certains obstacles à la promotion de l'autonomie ont été reliés aux caractéristiques de la clientèle, notamment aux valeurs et attentes spécifiques concernant les services infirmiers.

\section{Les facteurs personnels facilitants ou limitatifs}

De façon générale, les infirmières perçoivent que les programmes de maintien à domicile offrent un grand potentiel d'autonomie dans la pratique des soins infirmiers en congruence avec les valeurs de cette pratique professionnelle. Elles estiment en effet avoir plein pouvoir d'organiser leurs tâches, de décider de leurs priorités et des actions à poser dans I'exercice de leurs fonctions. Cette perception de grande autonomie est considérée comme étant un important facteur de motivation au travail.

Elles affirment valoriser l'approche globale et l'intervention éducative et de soutien auprès du bénéficiaire et de ses proches, et, de façon générale, estiment pouvoir appliquer ces concepts dans leur pratique quotidienne. Cette congruence possible entre leurs valeurs personnelles et l'action semble également être un facteur qui contribue à motiver les infirmières dans leur travail. Par exemple, l'importance de l'autonomie des bénéficiaires est une valeur que partagent les infirmières. Elles comparent leur rôle à celui d'une mère qui se réjouit de voir ses enfants se débrouiller dans la vie avec leurs propres moyens.

L'autonomie implique parfois de devoir mettre un terme à la relation infirmière-bénéficiaire. Cette forme de « rupture » requiert une certaine forme $d^{\prime}$ amour, mais les infirmières considèrent que pour soigner les gens à domicile cet amour s'avère indispensable.

Je vois la relation d'un jeune enfant avec sa mère, c'est valorisant un bébé, parce que tu sens qu'il a besoin de toi. Mais à partir du moment où il commence à être autonome, tu vas l'aimer aussi ton enfant, tu vas sentir une valorisation parce que tu es capable, toi, de le rendre autonome. Je mets ça en parallèle parce que je pense que c'est un peu la même chose. Sentir que tu as eu confiance en la personne, que la personne a eu confiance en toi et qu'elle a été capable d'apprendre, qu'elle était disponible pour apprendre. Tu l'as soutenue, et puis à un moment ou l'autre, elle $\mathrm{s}^{\prime}$ en va toute seule. Je pense que c'est la même satisfaction. Oui, il faut aimer beaucoup. Je pense que la base des soins avec les personnes, c'est l'amour. Tu peux pas t'en tirer sans ça. On aime les gens et les gens nous aiment aussi. C'est un amour qui est partagé, qui est réciproque. Je pense que la vie a du sens et de I'intérêt quand il y a de l'amour. 
Mais, comme nous le rappellent Guberman, Maheu et Maillé (1991), l'amour ne suffit pas toujours! Les infirmières estiment qu'elles manquent de formation plus spécialisée en relation d'aide et que cette lacune serait parfois à l'origine d'une incapacité à cerner certaines problématiques plus complexes liées à la promotion de l'autonomie, surtout dans le contexte de l'alourdissement des clientèles, tant du point de vue du nombre que des caractéristiques des bénéficiaires. À titre d'exemples, mentionnons les situations où à l'occasion de la prestation d'un soin technique (ex.: changer un pansement) I'infirmière doit faire face à un problème de violence conjugale. Le climat de confiance créé par l'infirmière entraîne l'expression de problèmes plus complexes, telles l'impuissance ressentie par les femmes devant ces situations, la perte de l'estime de soi, etc. Bien que ces situations nécessitent une intervention plus spécialisée, le plus souvent de la part d'un travailleur social, les infirmières doivent souvent intervenir au premier plan avant que les personnes concernées acceptent l'intervention d'un autre professionnel. Le refus de traitement ou de collaboration, les problèmes de santé mentale tels que les dépressions sont aussi des exemples de situations complexes auxquelles les infirmières sont confrontées et qui nécessitent des compétences en relation d'aide.

Les infirmières reconnaissent le soutien apporté par le travail multidisciplinaire pour la solution de problèmes complexes, notamment l'effet positif de la présence du coordonnateur ou de la coordonnatrice au moment des rencontres d'équipes. Leur présence permet une prise de conscience du rôle parfois "élargi » de l'infirmière, qui doit accroître le temps consacré à l'écoute et aux interventions éducatives. Il devient alors plus facile pour celle-ci de «négocier» une plus grande disponibilité auprès de la clientèle qui vit des situations complexes.

Par ailleurs, de plus en plus, des modèles conceptuels de soins infirmiers viennent orienter les démarches de soins infirmiers qui s'implantent ou qui sont en voie d'implantation. Au moment de nos rencontres, plusieurs des CLSC intégraient un modèle conceptuel de soins infirmiers à leur milieu, de manière que, dans le futur, les plans de soins puissent se baser sur ces modèles, favorisant entre autres l'établissement des priorités de soins et des priorités d'interventions qui visent l'autonomie. L'utilisation de ces outils de travail est censée permettre aux infirmières d'établir plus facilement les priorités d'intervention.

\section{Les facteurs organisationnels facilitants ou limitatifs}

Le fait pour les infirmières de travailler directement auprès du bénéficiaire, en association avec les proches, constitue un élément d'intérêt pour la promotion de l'autonomie en soins à domicile. Cette complicité qu'elles établissent avec les familles allègerait en quelque sorte 
la tâche, puisque les besoins décroîtraient en proportion de la participation de ces dernières.

Le fait que la voie d'accès au bénéficiaire soit le «corps» dans son contexte familier et naturel confère aux situations de soins un caractère d'intimité qui est perçu comme un élément facilitateur de la création d'une relation significative.

Prodiguer des soins dans le milieu de vie des personnes amène un sentiment d'être "attendue » et "désirée " par les bénéficiaires. $L^{\prime}$ 'infirmière a de cette manière un sentiment $d^{\prime}$ 'utilité et ressent comme un privilège la position de confidente, devenant même, dans certains cas, elle-même un membre significatif du réseau des bénéficiaires et des familles. Cette intimité apparaît donc comme un autre aspect de la motivation des infirmières dans les services de soutien à domicile.

Ça nous amène aussi une satisfaction d'être utile, je veux dire que c'est valorisant. On a l'impression, quand on entre là, qu'on est quasiment le bon Dieu pour eux. On a l'impression qu'on leur apporte quelque chose, $c^{\prime}$ est un sentiment qui est valorisant, on a l'impression de donner... C'est leur feed-back: quand on entre chez les gens, on voit qu'ils sont tout tristes, et quand on part, on les regarde, et ils sont contents: « Vous m'avez fait tellement de bien. » Ça fait du bien, ça, c'est valorisant.

Les infirmières estiment toutefois qu'il y a parfois un manque de concertation dans l'évaluation des besoins des services infirmiers à domicile. Par exemple, elles ont mentionné des situations où le médecin décide d'offrir des services à des personnes uniquement en fonction d'un critère d'âge, alors que la personne serait assez autonome pour se déplacer ou pour participer plus activement aux soins que requiert son état de santé.

Elles considèrent aussi que certaines croyances et valeurs de la clientèle constituent fréquemment un obstacle à la promotion de l'autonomie. II semble notamment que ce sont en général les personnes les plus à l'aise financièrement qui réclament le plus les services du CLSC et ce, sans que leur niveau d'autonomie l'exige nécessairement. Ces personnes demandent les services en alléguant que leur âge (65 ans, âge de la retraite) justifie leur droit aux services infirmiers à domicile. $\mathrm{D}^{\prime}$ autres personnes estiment que le fait $d^{\prime}$ avoir payé des taxes toute leur vie leur donne d'emblée un droit à des services à domicile et à des services infirmiers. C'est le cas notamment des personnes qui refusent de se rendre dans une clinique privée ou une clinique externe pour des examens de laboratoire, ou des personnes qui refusent de collaborer à certains soins tels que l'administration d'injections ou le changement de pansements, etc.

Il y a peut-être aussi le fait que les gens qui se retrouvent dans ces résidences et qui sont des gens financièrement plus à l'aise 
connaissent mieux le système. Les gens plus riches sont plus exigeants. En tout cas pour cette catégorie-là de gens âgés... Je pense que les personnes âgées qui sont à l'aise financièrement sont beaucoup plus exigeantes.

Certains clients croient qu'il est convenable de consulter différents médecins en plus de leur médecin de famille afin d'obtenir une ordonnance de médicaments ou de services à domicile. Ce phénomène semble plus courant en résidence pour personnes âgées où il y a visites systématiques de médecins. Il semble également que dans ces milieux on observe parfois une certaine fausse représentation des services disponibles et que, en conséquence, les bénéficiaires se tournent vers les CLSC pour obtenir les services annoncés par ces résidences.

D'autres clients croient que, puisque le MSSS accroît le budget des services à domicile, il y aurait ainsi une plus grande disponibilité des services infirmiers pour chaque personne. On observe donc, jusqu'à un certain point, une méconnaissance du fonctionnement des services de santé et des ressources disponibles.

C'est peut-être la manière dont c'est présenté au niveau politique. On veut maintenir les gens à domicile, donc on leur donne ceci, on leur donne cela. Quand on arrive dans la réalité, c'est pas vrai: on n'a pas les budgets, et on ne leur donne pas ça.

Ainsi, il semble y avoir un écart entre les politiques gouvernementales de maintien à domicile et la perception des clients. Les infirmières observent en effet que cette politique est davantage interprétée comme un droit accru aux services à domicile. Les bénéficiaires ne semblent pas percevoir une incitation au maintien de l'autonomie individuelle et familiale, ou une invitation à une plus grande participation des citoyens dans la solution de leurs problèmes ou dans les soins que nécessite leur état de santé.

\section{LES ENJEUX SOCIAUX DE LA PRATIQUE DES SOINS INFIRMIERS À DOMICILE}

De la description qui précède, quels enjeux sociaux pouvons-nous dégager pour la pratique des soins infirmiers à domicile? Par rapport à des dimensions comme l'autonomie et le partenariat, qui sont au cœur de la philosophie des services de soutien à domicile en CLSC, devant quels constats et interrogations nous trouvons-nous ici?

Voyons d'abord la question de l'autonomie. Nous avons vu que les soins infirmiers à domicile nécessitent une définition nuancée de I'autonomie, c'est-à-dire non universelle. Dans le quotidien des soins, I'autonomie est certes un idéal à atteindre, mais les situations de soins 
ne sont pas toujours favorables à son expression ou encore à son développement. Les soignantes et soignants sont amenés à tenir compte des limitations et capacités des personnes en respectant leur rythme, leur âge et leur condition. Les situations de soins, peut-être plus que $\mathrm{d}$ 'autres, nous ramènent là où est le corps, dénué de toute abstraction. Lorsque des situations de santé exigent que I'on prenne soin de son corps plusieurs heures par jour, les infirmières font face à des réalités bien éloignées de la rationalité étatique. Qu'est-ce que I'autonomie dans le soutien à domicile? Est-ce que l'autonomie se limite ici à la capacité de rester chez soi, de s'auto-soigner, tout en requérant, quelques heures par semaine, des soins professionnels? L'autonomie constitue en fait une réalité virtuelle, et il est à se demander dans quelle mesure il ne faudrait pas relier sa définition à celle de la qualité de vie.

En posant l'autonomie comme associée à un contexte, les infirmières ont remis en question l'universalité des valeurs de la réforme. Tout en acceptant l'autonomie en tant que valeur, celle-ci ne saurait être exportée de manière uniforme à toutes les catégories de clientèle ; à ce chapitre, on ne saurait confondre valeur et norme, le souhaitable et l'obligatoire. Si tous ne sont pas égaux devant la santé, de même, tous ne sont pas égaux devant l'autonomie. Aussi le citoyen lui-même peut-il invoquer son «droit aux services» malgré tout ce qu'on lui retire ou menace de lui retirer. Devant la perte d'autonomie, certains s'attendent à des compensations, à des soins. Si les technocrates ont interprété le refus de l'institutionnalisation comme une autorisation à limiter les services, il n'en va pas de même nécessairement chez ceux qui choisissent de demeurer à domicile. Et l'on revient dans le champ des valeurs. Qui est ce citoyen au centre des services à qui on fait référence dans la réforme? De quoi décide-t-il? Que devient-il lorsqu'il est malade à domicile et qu'il requiert des soins? Qui parle en son nom de son autonomie? L'un des enjeux sociaux des soins consiste à ce que l'autonomie demeure une valeur et un idéal, et ne se transforme pas en une norme, ce qui nous guette dans le contexte que nous connaissons.

Réfléchissons maintenant à la question du partenariat. On parle partout de partenariat. Tout le système de santé doit se penser en partenariat avec le «milieu naturel ». On a déjà noté les dangers de cette fausse relation partenaire, où l'une des parties (I'État, le secteur formel, l'intervenant) définit le sens du partenariat, et où l'autre partie (le milieu naturel, le secteur informel, le bénéficiaire) se fait l'appendice de la première (Panet-Raymond et Bourque, 1991). Cette observation, fondée, ne signifie pas pour autant que l'idée de partenariat soit inintéressante. La valorisation de I'autonomie et du partenariat est née, il faut se le rappeler, de la critique du professionnalisme (Levin, 1976). Dans leurs discussions, les infirmières ont insisté sur l'importance de 
I'individualisation de la relation de soins; elles nous rappellent que, dans le contexte des soins à domicile, dans nombre de cas il est possible $d^{\prime}$ 'atteindre l'autonomie en étant en véritable relation avec les personnes. Elles ont en fait reconnu le caractère dialogique de la relation de soins (Benner et Wrubel, 1989). Le partenariat se concrétise dans un contrat, à portée symbolique, que l'on défait et refait dans le temps, quand chacun «fait son bout de chemin ». C'est dans cet espace que se négocie le "partage des responsabilités » entre les différents acteurs du soutien à domicile. Y a-t-il des contours à ce partage? On connaît encore trop peu de cet univers de négociations des responsabilités de soins; il y a là une voie de choix pour comprendre "de l'intérieur» la réalité du partenariat. Dans quel contexte juge-t-on de la nonnécessité des soins professionnels? de leur exigence? On a jusqu'à maintenant beaucoup épilogué sur le fardeau des soins, sur sa mesure, notamment dans le réseau informel. Le partenariat implique deux parties, il nécessite relation et négociations, il vit dans un processus. La véritable question qui se pose est peut-être celle-ci : jusqu'à quel point vise-t-on non pas le partenariat mais plutôt le transfert des responsabilités? Le partenariat nous renvoie à l'échange social; le transfert nous entraîne dans le domaine de l'imputabilité, puis de la délégation. Cela représente un autre enjeu social majeur, dont les soins se trouvent révélateurs (Boucher-Dancause, 1994).

Enfin, I'alourdissement et la complexification des situations de soins observés par les infirmières nécessitent des outils pour faire face aux besoins nouveaux. Les analyses féministes ont montré que les soins sont souvent relégués dans la sphère du privé, du relationnel et de l'affect (Saillant, 1991 et 1992). Trop souvent considère-t-on les activités de soins comme faisant partie des qualités naturelles et attendues des femmes; est-ce pour cette raison que l'on pense plus facilement à les déléguer et à les déprofessionnaliser? Quoi qu'il en soit, la recherche des outils dans le domaine des soins à domicile passe notamment par une meilleure définition de la pratique, par sa reconnaissance dans le contexte de la multidisciplinarité, et par la visibilité sociale. Une meilleure définition des soins et des soins infirmiers à domicile permettrait une compréhension accrue du champ de pratique qu'est le soutien à domicile, compte tenu de la place qu'ils y occupent.

\section{CONCLUSION}

Cette étude exploratoire de la pratique des soins infirmiers à domicile dans les CLSC de la région de Québec nous a permis de mieux connaître le contenu, le contexte et les enjeux sociaux de cette pratique. 
Cette étude est un premier pas pour mieux définir les soins infirmiers à domicile par rapport à la promotion de l'autonomie par le partenariat. Elle a été menée auprès d'un groupe d'infirmières volontaires dont le rôle était de faire ressortir les caractéristique de leur pratique professionnelle. L'analyse du contenu des entrevues de groupe révèle une pratique de soins infirmiers visant à répondre à des besoins très diversifiés chez la clientèle. Les soins infirmiers reliés à la promotion de I'autonomie doivent s'adapter au potentiel d'autonomie et aux divers contextes de vie des personnes et des familles. Les situations de soins sont par ailleurs très variées et certains facteurs personnels et organisationnels contribuent à faciliter ou à limiter la prestation des soins visant la promotion de l'autonomie. Nous avons relevé des nuances importantes en ce qui concerne les construits d'autonomie et de partenariat qui ne peuvent s'appliquer de manière universelle aux diverses situations de soins. Les données de cette étude se limitent à un nombre restreint d'infirmières représentant des CLSC d'une région du Québec. Quoique non généralisables à l'ensemble des pratiques professionnelles de soins infirmiers des programmes de maintien à domicile des CLSC, ces résultats nous permettent cependant de franchir une première étape dans la description et l'analyse d'un univers de pratiques dont la nature et la contribution sont souvent méconnues.

À l'instar de tous les autres intervenants des CLSC, les infirmières doivent contribuer à la réalisation de la mission et des objectifs des CLSC. Le maintien ou la promotion de l'autonomie de la clientèle constituent à cet égard un des enjeux majeurs des programmes de maintien à domicile. Ces services sont largement multidisciplinaires et constituent de ce fait un défi considérable lorsqu'il s'agit de bien définir la spécificité du champ d'intervention de chaque type d'intervenants. Dans quelle mesure et par quelles stratégies d'action les infirmières contribuent-elles à maintenir les personnes dans leur milieu de vie tout en assurant la sécurité et un état de bien-être et de santé optimal selon la situation des usagers?

La réponse à cette question est ici incomplète et sera davantage fouillée dans une étude plus vaste à venir. Par ailleurs, les enjeux sociaux des soins sont tels qu'ils nous amènent à nous interroger sur les conceptions actuellement en usage dans l'univers des pratiques du soutien à domicile sur le sens de l'autonomie, du partenariat et des soins infirmiers. 


\section{Références bibliographiques}

ACREE, E.L. et V.J. GREGORY (1989). "Home Health Care Through an Academic Nurse-Managed Center », National League for Nursing, 21-2311 : 135-141.

BENNER, P. et J. WRUBEL (1989). The primacy of caring in health and illness. Don Mills (Ont.) : Addison-Wesley Publ., Co.

BOUCHER-DANCAUSE, G. (1994). Étude sur le contenu et la signification du transfert des responsabilités de soins infirmiers aux personnes âgées dans le cadre d'un programme de maintien à domicile de deux CLSC de la région de Québec. Mémoire de maîtrise en sciences infirmières. SainteFoy: Université Laval.

COLLIÈRE, M.F. (1986). "L'avenir du soutien à domicile et son financement. La complémentarité des actions - Les soins dans le soutien à domicile », Soins, $\mathrm{n}^{\circ}$ 474, mars : 36-40.

FÉdÉRATION DES CLSC DU QUÉBEC (1991). Document de réflexion $\mathrm{n}^{\circ} 4$ : Le soutien à domicile. Avril.

GARANT, L. et M. BOLDUC (1990). L'aide par les proches: mythes et réalités. Gouvernement du Québec: MSSS.

GiLmORE, G.D., M.D. CAMPBELL et B.L. BeCKER (1989). Needs assessment strategies for health education and health promotion. Indianapolis (Indiana): Benchmark, p. 69-73.

GLAZER, N.Y. (1992). "The home as workshop: women as amateur nurses and medical care providers", Gender and Society, vol 14, n $4: 479-499$.

GuBERMAN, N., P. MAHEU et C. MAILlÉ (1991). Et si l'amour ne suffisait pas. Montréal: Les Éditions du Remue-Ménage.

HAGAN, L. (1991). "L'éducation pour la santé en CLSC», Nursing Québec, vol. $11, \mathrm{n}^{\circ} 4$, juillet/août.

HAGAN, L., F. SAILLANT et G. DANCAUSE (1994). «Maintien à domicile et promotion de l'autonomie des clientèles: vers une meilleure connaissance de I'enjeu pour la pratique des soins infirmiers dans les programmes de soutien à domicile des CLSC », L'infirmière du Québec, vol. 1, nº 5 : 35-41.

HAMILTON, D. (1988). "Clinical Excellence, but Too High a Cost: The Metropolitan Life Insurance Company Visiting Nurse Service (1909-1953)», Public Health Nursing, vol. 5, $\mathrm{n}^{\circ}$ 4, déc. : 235-240.

HORNING, J.C. (1991). "Support and Education for the Caregiver and Client in the Home: An Inservice Project for Community Health Nurses », Journal of Community Health Nursing, vol. 8, $\mathrm{n}^{\circ} 3$ : 155-161.

HUMPHREY, C.J. (1988). "The home as a setting for care. Clarifying the boundaries of practice», Nursing Clinics of North America, vol. 23, $\mathrm{n}^{\circ} 2$, juin : 305-314.

JOHNSON, E.A. et J.E. JACKSON (1989). "Teaching the Home Care Client», Nursing Clinics of North America, vol. 24, $\mathrm{n}^{\circ} 3$, septembre : 687-693.

JUTRAS, S. et M. RENAUd (1987). Personnes âgées et aidants naturels. Éléments pour une réflexion sur la prévention dans le plan d'ensemble "la santé pour tous ". Montréal : Université de Montréal, GRASP.

JUTRAS, S. et F. VEILLEUX (1989). Des partenaires méconnus: les aidants des personnes âgées en perte d'autonomie. Montréal : Université de Montréal, GRASP. 
KERKSTRA, A. et al. (1991). "Preventive home visits to elderly people by community nurses in The Netherlands », Journal of Advanced Nursing, 16 : 631-637.

KRISTJANSON, L. et K. CHALMERS (1990). "Nurse-Client Interactions in Community-Based Practice: Creating Common Ground», Public Health Nursing, vol. 7, $\mathrm{n}^{\circ}$ 4, déc. : 215-223.

LeSEMANN, F. et F. Chaume (1989). Familles-providence. La part de l'État. Groupe d'analyse des politiques sociales. Université de Montréal, Montréal: Les Editions St-Martin.

LEVIN, L.S. et al. (1976). Self Care, Lay Initiatives in Health. New York: Prodist.

MCMAHON, R. (1990). "Partners in Care», Nursing Times, vol. 85, n 8, 22 février: 34-37.

MINISTÈRE DE LA SANTÉ ET DES SERVICES SOCIAUX (1990). Une réforme axée sur le citoyen. Québec.

PANet-RAymOnd, J. et D. BOURQue (1991). Partenariat ou pater-nariat?, Rapport de recherche, Université de Montréal, École de service social.

PESZNECKER, B.L. et al. (1989). "The Mutual-Participation Relationship: Key to Facilitating Self-Care Practices in Clients and Families », Public Health Nursing, vol. 6, $\mathrm{n}^{\circ} 4: 197-203$.

RoY, J., A. VÉZINA et M. PARADIS (1992) De la tâche à la mission. Personnes âgées, aidants naturels, services intensifs de maintien à domicile (SIMAD). Centre de recherche sur les services communautaires. SainteFoy: Université Laval.

ROY, J., F. SAILLANT et A. VÉZINA (1994). Priorités de recherche en soutien à domicile. Bilan des consultations en CLSC. Centre de recherche sur les services communautaires, Sainte-Foy: Université Laval.

SAILLANT, F. (1992). "La part des femmes dans les soins de santé », Revue internationale d'action communautaire, 28/68: 95-106.

THERRIEN, R. (1987). La contribution informelle des femmes aux services de santé et aux services sociaux. Québec: Les Publications du Québec.

VAN AGthoven, W.M. et H.N. Plomp (1989). "The interpretation of self-care: a difference in outlook between clients and home-nurses», Social Science and Medicine, vol. 29, $\mathrm{n}^{\circ} 2: 245-252$.

WHALL, A.L. (1986). "The Family as the Unit of Care in Nursing: A Historical Review», Public Health Nursing, vol. 3, n 4 : 240-249.

WILES, E. (1984). "Home health care nursing», dans M. STANHOPE et J. LANCASTER, Community health nursing. Process and practice for promoting health. Toronto: Mosby Co., p. 780-804. 